RODRIGUES, S. R.; PEREIRA, A. F. Scarabaeidae pragas em sucessão de soja e algodão em Campo Novo dos Parecis, MT. Revista de Agricultura Neotropical, Cassilândia-MS, v. 1, n. 1, p. 38-43, jul./set. 2014.

\title{
SCARABAEIDAE PRAGAS EM SUCESSÃO DE SOJA E ALGODÃO EM CAMPO NOVO DOS PARECIS, MT
}

\section{SÉRGIO ROBERTO RODRIGUES ${ }^{1}$, ALEX FURQUIM PEREIRA ${ }^{1}$}

\footnotetext{
${ }^{1}$ UEMS/Cassilândia, MS, sergio@uems.br, alex_furquim@hotmail.com
}

\begin{abstract}
RESUMO: O Estado de Mato Grosso possui as maiores áreas cultivadas com soja e algodão e face às extensas áreas cultivadas várias são as espécies de pragas que ocorrem, incluindo os coleópteros fitófagos da família Scarabaeidae. Assim, foram desenvolvidos estudos para conhecer as espécies de Scarabaeidae ocorrentes no sistema de sucessão de cultura de soja e algodão em Campo Novo dos Parecis, MT. Para obtenção dos imaturos foram realizadas amostragens em janeiro de 2011, na cultura da soja, em abril na cultura do algodão, em julho quando a área estava em pousio e na cultura da soja em dezembro. Em 2012, as amostragens foram realizadas em janeiro na cultura da soja e em fevereiro e março na cultura do algodão. As larvas amostradas foram levadas para o laboratório de entomologia da Universidade Estadual de Mato Grosso do Sul, e criadas até atingirem a fase adulta. Em janeiro, abril, julho e dezembro de 2011, coletaram-se respectivamente, 1,0, 0,0, 0,0 e 0,7 larvas $\mathrm{m}^{-2}$ e em janeiro, fevereiro e março de 2012, 1,0, 0,0 e 0,0 larvas $\mathrm{m}^{-2}$, respectivamente. Os adultos foram identificados como Leucothyreus sp e Leucothyreus aff. ambrosius. Nos estudos realizados na sucessão de soja e algodão, em Campo Novo dos Parecis, Leucothyreus se caracteriza como o principal gênero de Scarabaeidae praga.
\end{abstract}

PALAVRAS-CHAVE: Coleoptera, Geniatini, pragas de solo, imaturos, grandes culturas.

\section{SCARABAEIDAE PESTS IN SUCCESSION OF SOYBEAN AND COTTON IN CAMPO NOVO DOS PARECIS}

\begin{abstract}
The state of Mato Grosso has the largest planted area with soybeans and cotton, and given the extensive acreages there are several species of pests that occur, including phytophagous beetles from the Scarabaeidae family. Thus, studies were undertaken to know the occurring species of Scarabaeidae in the soybean and cotton succession system in Campo Novo dos Parecis, Mato Grosso state. In order to obtain the immature samples were performed in January 2011, in soybean, in April in cotton, in July when the areas were fallow and soybean in December. In 2012, sampling was conducted in January in soybeans and in February and March in cotton. The sampled larvae were taken to the entomology laboratory at the State University of Mato Grosso do Sul, and reared until they reach adulthood. In January, April, July and December 2011, it was respectively collected 1.0, 0.0, 0.0 and 0.7 larvae $\mathrm{m}^{-2}$ and in January, February and March 2012, the sample yielded 1.0, 0.0 and 0.0 larvae $\mathrm{m}^{-2}$, respectively. The adults were identified as Leucothyreus sp and Leucothyreus aff. ambrosius. In studies conducted in succession of soybean and cotton, in Campo Novo dos Parecis, the Leucothyreus is characterized as the main genera of Scarabaeidae pest.
\end{abstract}

KEY WORDS: Coleoptera, Geniatini, soil pests, immature, field crops. 


\section{INTRODUÇÃO}

O Estado de Mato Grosso possui as maiores áreas de soja e algodão cultivadas no Brasil (CONAB, 2014). Normalmente as pragas que aparecem na parte aérea dessas culturas são bem conhecidas, entretanto, as pragas que permanecem no solo e ai se desenvolvem, são pouco estudadas. Um importante grupo de pragas de solo são os coleópteros da família Scarabaeidae, os quais aparecem associados a várias plantas cultivadas (COUTINHO et al., 2011; RODRIGUES et al., 2011; CHERMAN et al., 2013). Algumas espécies de Scarabaeidae, principalmente na sua fase imatura se alimentam de raízes de várias espécies vegetais, e ao se nutrirem de plantas cultivadas ocasionam prejuízos (SILVA, 1997; OLIVEIRA et al., 2004), uma vez que, provocam murcha, amarelecimento e posterior morte das mesmas.

Em algumas regiões do Brasil, foram relacionadas espécies de Scarabaeidae ocasionando danos à cultura da soja. No gênero Phyllophaga é relacionado $P$. cuyabana Moser, 1918 causando prejuízos em lavouras de soja no Estado do Paraná (OLIVEIRA et al., 2004), enquanto que $P$. triticophaga Morón \& Salvadori, 1998 é relacionado causando prejuízos em culturas no Rio Grande do Sul (SALVADORI; SILVA, 2004). Na cultura da soja em área de Cerrado no Distrito Federal foi encontrado P. aff. capillata Blanchard, 1850 causando danos (OLIVEIRA et al., 2007a). Em Mato Grosso do Sul, foi relacionada à ocorrência de Cyclocephala forsteri Endrodi, 1963 se desenvolvendo na cultura da soja (SANTOS; ÁVILA, 2007). Rodrigues et al. (2011) relacionaram Liogenys fusca Blanchard, 1851 como a principal espécie de Scarabaeidae praga associada a algumas culturas, incluindo a cultura da soja.

Na cultura do algodão, escassas são as informações sobre as espécies de Scarabaeidae associadas. Segundo Rodrigues et al. (2011) foram encontradas larvas de L. fusca associadas ao sistema radicular do algodão, porém em pequena quantidade. Oliveira et al. $(1997 ; 2004)$ informam que $P$. cuyabana se desenvolve na cultura do algodão, porém essa cultura não é o hospedeiro adequado.

Diante da grande expansão agrícola na região centro oeste, principalmente com as culturas de soja e algodão, foram desenvolvidos estudos para identificar as espécies de Scarabaeidae ocorrentes no sistema de sucessão de cultura de soja e algodão em Campo Novo dos Parecis, MT.

\section{MATERIAL E MÉTODOS}

Para realizar o estudo dos Scarabaeidae praga, foram realizadas coletas em área de culturas, na fazenda Bom Jesus, localizada a aproximadamente $35 \mathrm{~km}$ do município de

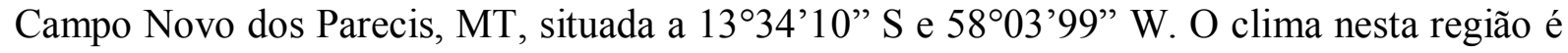
classificado como Aw, segundo a classificação de Köppen-Geiger, com chuvas no verão e inverno seco, precipitação variando de 1.500 a $2.200 \mathrm{~mm}^{-1} \mathrm{ano}^{-1}$ e temperaturas médias anuais entre $21^{\circ} \mathrm{C}$ e $26,8^{\circ} \mathrm{C}$ (MOREIRA; VASCONCELOS, 2007).

Na fazenda Bom Jesus, o sistema de sucessão empregado é soja no verão e algodão na safrinha. Para desenvolvimento dos estudos foi utilizado um talhão com 150 ha (Figura 1). Na safra 2010/2011, a área amostrada, estava sendo cultivada com a variedade de soja, Monsoy 7639 RR e com a variedade de algodão IMACD 6001 LL e, na safra 2011/2012 com a variedade de soja Monsoy 7908 RR e de algodão IMACD 6001 LL.

Para obtenção das larvas de Scarabaeidae, associadas às culturas, foram realizadas amostragens em janeiro de 2011, na cultura da soja, em abril na cultura do algodão, em julho 
quando as áreas de cultivo estavam em pousio e novamente na cultura da soja em dezembro. Em 2012, as amostragens foram realizadas em janeiro na cultura da soja e em fevereiro e março na cultura do algodão. Nestas culturas, em cada mês de amostragem foram feitas aleatoriamente, 50 trincheiras na linha de semeadura das culturas, com dimensões de $25 \mathrm{x} 25 \mathrm{x}$ $30 \mathrm{~cm}$ de profundidade, sendo possível a visualização e extração das larvas de Scarabaeidae (metodologia adaptada de PARDO-LOCARNO et al., 2005; COUTINHO et al., 2011).

As larvas amostradas foram levadas para o laboratório de Entomologia da Universidade Estadual de Mato Grosso do Sul, campus de Aquidauana, onde foram mantidas, individualmente em recipientes de plástico de $500 \mathrm{ml}$, e criadas até atingirem a fase adulta. Nos recipientes de plástico, cerca de $2 / 3$ de seu volume foi preenchido com solo, juntamente com mudas de Brachiaria decumbens Stapf cv. Basilisk, até as larvas atingirem a fase de prépupa, sendo as mudas substituídas quinzenalmente por mudas novas, fornecendo desta maneira raízes para alimentação das larvas.
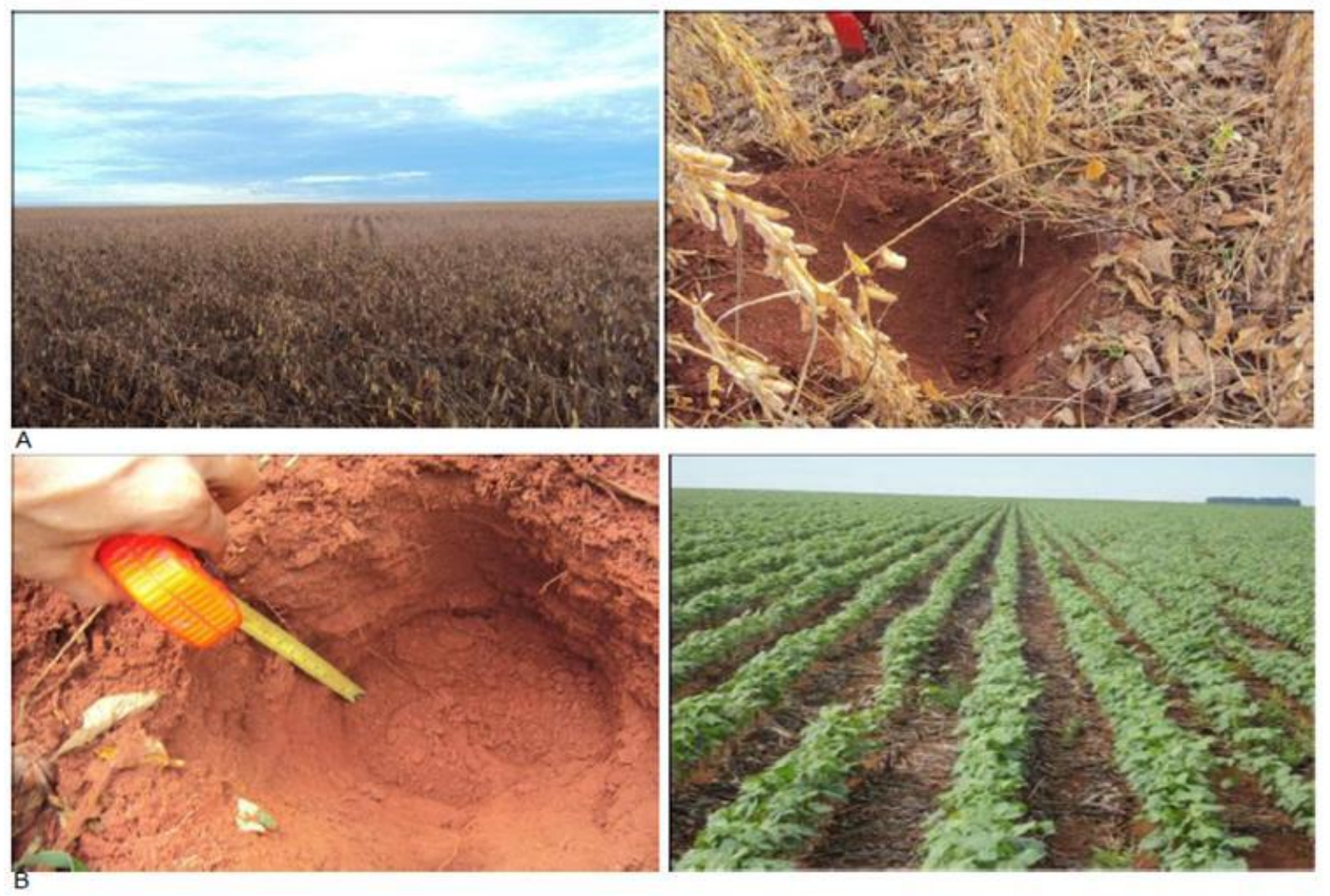

Figura 1. Áreas de amostragem da cultura da soja (A) e da cultura do algodão (B) em Campo Novo dos Parecis, MT.

Os adultos obtidos foram montados e depositados na coleção de entomologia da Universidade Estadual de Mato Grosso do Sul, em Aquidauana. A identificação foi realizada através da comparação com espécimes depositados na coleção da referida instituição, e também identificados pelo Dr. Miguel Angel Morón, Instituto de Ecologia, Xalapa, Veracruz, México.

\section{RESULTADOS E DISCUSSÃO}


As amostragens realizadas em janeiro, abril, julho e dezembro de 2011, originaram as densidades médias de 1,0, 0,0, 0,0 e 0,7 larvas $\mathrm{m}^{-2}$, respectivamente, e as amostragens realizadas em janeiro, fevereiro e março de 2012, as densidades médias de 1,0, 0,0 e 0,0 larvas $\mathrm{m}^{-2}$, respectivamente (Tabela 1 ).

Algumas informações são conhecidas sobre as densidades de larvas de Scarabaeidae em culturas, bem como seus níveis de danos. Silva e Costa (2002) ao estudarem as densidades larvais de Diloboderus abderus Sturm, 1826 em diversas culturas, verificaram que a população de 12 larvas $\mathrm{m}^{-2}$ em linho, 10 larvas $\mathrm{m}^{-2}$ em aveia preta, 0,5 larvas $\mathrm{m}^{-2} \mathrm{em}$ milho e 0,4 larvas $\mathrm{m}^{-2}$ em girassol, atingem o nível de dano econômico. Segundo Oliveira et al. (1997) para $P$. cuyabana populações médias de 20 larvas $\mathrm{m}^{-2}$, em soja, reduz a altura das plantas, tamanho dos grãos, número de vagens e número de grãos por planta, diminuindo em $50 \%$ a capacidade produtiva das plantas atacadas. Na cultura da cana-de-açúcar em Sidrolândia, MS, Coutinho et al. (2011) encontraram densidade média de larvas de Scarabaeidae, com 53,65 larvas $\mathrm{m}^{-2}$.

Nas amostragens realizadas na cultura da soja foram obtidas larvas de Scarabaeidae, enquanto que na cultura do algodão e pousio não foram coletadas larvas. Pode-se considerar que as densidades de larvas de Scarabaeidae, amostradas no sistema de sucessão de soja e algodão em Campo Novo dos Parecis, MT, são baixas, diante de densidades de larvas encontradas por outros pesquisadores em diferentes culturas, ou mesmo na cultura da soja em outras localidades.

Tabela 1. Densidade média de larvas por metro quadrado de Scarabaeidae amostrados em culturas de soja, algodão e pousio em Campo Novo dos Parecis, MT, em janeiro, abril, julho e dezembro de 2011 e, em janeiro, fevereiro e março de 2012.

\begin{tabular}{|c|c|c|c|c|c|c|c|}
\hline \multirow{3}{*}{ Culturas } & \multicolumn{6}{|c|}{ Densidades de larva $\mathrm{m}^{-2}$} & \\
\hline & \multicolumn{4}{|c|}{2011} & \multicolumn{3}{|c|}{2012} \\
\hline & Janeiro & Abril & Julho & Dezembro & Janeiro & Fevereiro & Março \\
\hline Soja & 1,0 & - & - & 0,7 & 1,0 & - & - \\
\hline Algodão & - & 0,0 & - & - & - & 0,0 & 0,0 \\
\hline Pousio & - & - & 0,0 & - & - & - & - \\
\hline
\end{tabular}

$\mathrm{Na}$ área onde se desenvolveu os estudos, as baixas densidades de larvas encontradas, podem estar sendo influenciadas pela presença da cultura do algodão. Segundo Macedo et al. (2007) em plantas de algodão há uma série de aldeídos-terpenos, como o gossipol, heliocidas e hemigossipolone, que funcionam como defesa contra várias espécies de insetos. Diante dessa informação, é possível entender que esses compostos produzidos pelas plantas de algodão, possam estar interferindo no ciclo biológico dos Scarabaeidae praga, amostrados em Campo Novo dos Parecis, MT.

A presença e densidade de larvas de Scarabaeidae em culturas de algodão já foram relatadas em alguns trabalhos científicos. Oliveira et al. (2007b) verificaram que fêmeas de $P$. cuyabana ovipositam menos, quando estão próximas à cultura do algodão. Menores densidades de larvas de Scarabaeidae foram obtidas por Rodrigues et al. (2011) em sucessões de cultura que possuíam plantas de algodão, quando comparado com outros sistemas de sucessão de culturas.

No sistema de sucessão de culturas, soja e algodão, além de se obter pequena densidade 
de larvas, houve diminuição na densidade de larvas da cultura de soja para a de algodão e, para o pousio. Assim, pode-se afirmar que a combinação da cultura de soja e algodão, não está sendo favorável ao desenvolvimento dos Scarabaeidae praga.

As larvas obtidas na cultura da soja após terem sido criadas em laboratório originaram adultos, que foram identificados como Leucothyreus sp e Leucothyreus aff. ambrosius. Assim, as espécies de Scarabaeidae pragas, pertencem ao gênero Leucothyreus. Foram amostradas larvas de Leucothyreus aff. ambrosius, em dezembro e janeiro, enquanto que Leucothyreus sp, foi amostrada em dezembro.

Algumas informações são conhecidas sobre a associação de espécies de Leucothyreus com plantas cultivadas. Segundo Pereira et al. (2013) larvas de L. alvarengai e L. aff. semipruinosus, desenvolvem-se em sistema de sucessão de soja e milho em Tangará da Serra, em Mato Grosso. Na Colômbia, adultos de Leucothyreus femoratus alimentam-se de folhas de Elaeis guineensis causando severa desfolha nas plantas (MARTINEZ et al. 2013).

\section{CONCLUSÕES}

O algodão em sucessão a soja, caracteriza-se como importante sistema que auxilia na redução de densidade de larvas de Scarabaeidae.

Leucothyreus é o principal gênero de Scarabaeidae praga na área estudada.

\section{AGRADECIMENTOS}

A Coordenação de Aperfeiçoamento de Pessoal de Nível Superior (CAPES), pela bolsa concedida a Alex Furquim Pereira. Ao Conselho Nacional de Desenvolvimento Científico e Tecnológico (CNPq), projeto “Casadinho" processo 620029/20080, pelo auxílio financeiro.

\section{REFERÊNCIAS BIBLIOGRÁFICAS}

CHERMAN, M. A.; GUEDES, J. V. C.; MORÓN, M. A.; DAL PRÁ, E.; BIGOLIN. M. White grubs (Coleoptera, Melolonthidae) in the "Planalto Region", Rio Grande do Sul state, Brazil: Key for identification, species richness and distribution. Revista Brasileira de Entomologia. Curitiba-PR, v. 57, n. 3, p. 271-278, 2013.

CONAB. COMPANHIA NACIONAL DE ABASTECIMENTO. Acompanhamento da safra brasileira de grãos. Safra 2013/14. n. 10. Décimo levantamento. Julho/2014. BrasíliaDF. $\quad$ v. $1, \quad$ p.1-85. $2014 . \quad$ Disponível em: http://www.conab.gov.br/OlalaCMS/uploads/arquivos/14_07_09_09_36_57_10_levantament o_de_graos_julho_2014.pdf Acesso em: 10 jul. 2014.

COUTINHO, G. V.; RODRIGUES, S. R.; CRUZ, E. C.; ABOT, A. R. Bionomic data and larval density of Scarabaeidae (Pleurosticti) in sugarcane in the central region of Mato Grosso do Sul, Brazil. Revista Brasileira de Entomologia, Curitiba-PR, v. 55, n. 3, p. 389-395, 2011.

MACEDO, L. P. M.; CUNHA, U. S.; VENDRAMIM, J. D. Gossipol: Fator de resistência a insetos-Praga. Campo Digital, Campo Mourão-PR, v. 2, n. 1, p. 34-42, 2007.

MARTÍNEZ, L. C.; PLATA-RUEDA, A.; ZANUNCIO, J. C.; SERRÃO, J. E. Leucothyreus femoratus (Coleoptera: Scarabaeidae): Feeding and behavioral activities as an oil palm defoliator. Florida Entomologist, Gainesville, Fla., v. 96, n. 1, p. 55-63. 2013. 
MOREIRA, M. L. C.; VASCONCELOS, T. N. N. Mato Grosso: solos e paisagens. $1^{\text {a }}$ Edição. Cuiabá-MT: Entrelinhas, 2007. 272 p.

OLIVEIRA, L. J.; GARCIA, M. A.; HOFFMANN-CAMPO, C. B.; FARIAS, J. R. B.; SOSAGOMEZ, D. R.; CORSO, I. C. Coró-da-soja Phyllophaga cuyabana. Londrina-PR: Embrapa CNPSo, 1997. 30 p. (Circular Técnica 20).

OLIVEIRA, C. M.; MORÓN, M. A.; FRIZZAS, M. R. First record of Phyllophaga sp aff. capillata (Coleoptera: Melolonthidae) as a soybean pest in the Brazilian "Cerrado". Florida Entomologist, Gainesville, Fla., v. 90, n. 4, p. 772-775, 2007 a.

OLIVEIRA, L. J.; GARCIA, M. A.; HOFFMANN-CAMPO, C. B.; AMARAL, M. L. B. Feeding and oviposition preference of Phyllophaga cuyabana (Moser) (Coleoptera: Melolonthidae) on several crops. Neotropical Entomology, Londrina-PR, v. 36, n. 5, p. 759764, $2007 \mathrm{~b}$.

OLIVEIRA, L. J.; SANTOS, B.; PARRA, J. R. P.; HOFFMANN-CAMPO, C. B. Coró-dasoja. In: SALVADORI, J. R.; ÁVILA C. J.; SILVA, M. T. B. Pragas de solo no Brasil. Passo Fundo: Embrapa Trigo; Dourados: Embrapa Agropecuária Oeste; Cruz Alta: Fundacep Fecotrigo, 2004, p. 167-190.

PARDO-LOCARNO, L. $\quad$ C.; MONTOYA-LERMA, J.; BELLOTTI, A. C.; SCHOONHOVEN, A. V. Structure and composition of the white grub complex (Coleoptera: Scarabaeidae) in agroecological systems of Northern Cauca, Colombia. Florida Entomologist, Gainesville, Fla., v. 88, n. 4, p. 355-363, 2005.

PEREIRA, A. F.; RODRIGUES, S. R.; MORÓN M.A. Biological aspects of Leucothyreus alvarengai Frey and Leucothyreus aff. semipruinosus Ohaus (Coleoptera, Melolonthidae, Rutelinae) in crop succession at central Brazil. Revista Brasileira de Entomologia, CuritibaPR, v. 57, n. 3, p. 323-328, 2013.

RODRIGUES, S. R.; CARMO, J. I.; OLIVEIRA, V. S.; TIAGO, E. F.; TAIRA, T. L. Ocorrência de larvas de Scarabaeidae fitófagos (Insecta: Coleoptera) em diferentes sistemas de sucessão de culturas. Pesquisa Agropecuária Tropical, Goiânia-GO, v. 41, n. 1, p. 87-93, 2011.

SALVADORI, J. R.; SILVA, M. T. B. Coró-do-trigo. In: SALVADORI, J. R.; ÁVILA C. J.; SILVA, M. T. B. Pragas de solo no Brasil. Passo Fundo: Embrapa Trigo; Dourados: Embrapa Agropecuária Oeste; Cruz Alta: Fundacep Fecotrigo, 2004, p. 210-232.

SANTOS, V.; ÁVILA, C. J. Aspectos bioecológicos de Cyclocephala forsteri Endrodi, 1963 (Coleoptera: Melolonthidae) no Estado do Mato Grosso do Sul. Revista de Agricultura, Piracicaba-SP, v. 82, n. 3, p. 298-303, 2007.

SILVA, M. T. B. Níveis de controle de Diloboderus abderus (Sturm) em trigo no plantio direto. Anais da Sociedade Entomológica do Brasil, Londrina-PR, v. 26, n. 3, p. 435-440, 1997.

SILVA, M. T. B.; COSTA, E.C. Nível de controle de Diloboderus abderus em aveia preta, linho, milho e girassol. Ciência Rural, Santa Maria-RS, v. 32, n. 1, p. 7-12, 2002. 